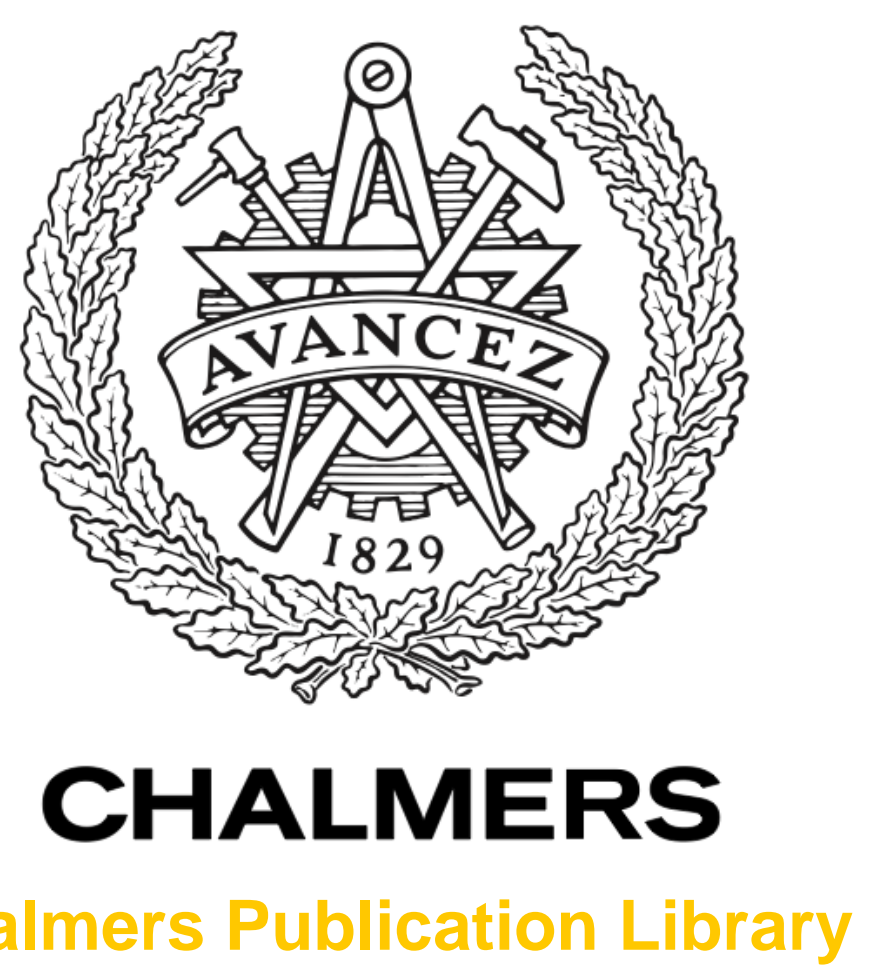

Chalmers Publication Library

\title{
Bandwidth scaling of a phase-modulated CW comb through four-wave mixing in a silicon nano-waveguide
}

This document has been downloaded from Chalmers Publication Library (CPL). It is the author's version of a work that was accepted for publication in:

Optics Letters (ISSN: 0146-9592)

Citation for the published paper:

Liu, Y. ; Metcalf, A. ; Torres Company, V. (2014) "Bandwidth scaling of a phase-modulated

$\mathrm{CW}$ comb through four-wave mixing in a silicon nano-waveguide". Optics Letters, vol.

39(22), pp. 6478-6481.

Downloaded from: http://publications.lib.chalmers.se/publication/208731

Notice: Changes introduced as a result of publishing processes such as copy-editing and formatting may not be reflected in this document. For a definitive version of this work, please refer to the published source. Please note that access to the published version might require a subscription.

Chalmers Publication Library (CPL) offers the possibility of retrieving research publications produced at Chalmers University of Technology. It covers all types of publications: articles, dissertations, licentiate theses, masters theses, conference papers, reports etc. Since 2006 it is the official tool for Chalmers official publication statistics. To ensure that Chalmers research results are disseminated as widely as possible, an Open Access Policy has been adopted.

The CPL service is administrated and maintained by Chalmers Library. 


\title{
Bandwidth scaling of a phase-modulated CW comb through four-wave mixing in a silicon nano-waveguide
}

\author{
Yang Liu, ${ }^{1 *}$ Andrew J. Metcalf, ${ }^{1}$ Victor Torres Company, ${ }^{1,2}$ Rui Wu,${ }^{1,3}$ Li Fan,${ }^{1,4}$ Leo T. Varghese,,${ }^{1,4}$ Minghao \\ $\mathrm{Qi},{ }^{1,4}$ and Andrew M. Weiner ${ }^{1,4}$ \\ ${ }^{1}$ School of Electrical and Computer Engineering, Purdue University, 465 Northwestern Avenue, West Lafayette, IN 47907-2035 \\ ${ }^{2}$ Current Address: Department of Microtechnology and Nanoscience (MC2), Chalmers University of Technology, 41296 \\ Gothenburg, Sweden \\ ${ }^{3}$ Current Address: Intel Corporation, 2501 NW 229th Avenue, Hillsboro, OR 97124 \\ ${ }^{4}$ Birck Nanotechnology Center, Purdue University, 1205 West State Street, West Lafayette, Indiana 47907, USA \\ *Corresponding author: yangliu@purdue.edu
}

\begin{abstract}
We demonstrate an on-chip four-wave-mixing (FWM) scheme in a silicon nano-waveguide to scale the bandwidth of a frequency comb generated by phase modulation of continuous-wave $(\mathrm{CW})$ lasers. The FWM process doubles the bandwidth of the initial comb generated by the modulation of a $\mathrm{CW}$ laser. As an example, a wavelength-tunable frequency comb with $>100 \mathrm{comb}$ lines spaced by $10-\mathrm{GHz}$ within $5-\mathrm{dB}$ bandwidth is generated. OCIS codes: (190.4390) Nonlinear optics, integrated optics; (060.5060) Phase modulation; (060.5625) Radio frequency photonics
\end{abstract}

Strong sinusoidal phase modulation of a continuous-wave (CW) laser can create multiple sidebands to form a frequency comb $[1,2]$. This technique enables the creation of highrepetition-rate combs with stable optical frequencies while allowing for independent tuning of the repetition rate and optical center frequency. These attributes, combined with the simplicity of the technique, make it an ideal candidate for applications in optical communications [3], radio-frequency (RF) photonics [4] and optical arbitrary waveform generation (OAWG) [5]. By phase modulation (PM) alone, the spectral lines suffer from significant line-to-line amplitude variations, i.e. poor spectral flatness. The spectral flatness of the comb can be improved considerably by adding an intensity modulator (IM) in series with the PM [6, 7]. In this scheme, the IM acts to carve out a flat-topped pulse train from the $\mathrm{CW}$ source, whereas the phase modulator introduces quadratic phase on each pulse (thus acting as a time-lens [8]). When correctly aligned, the carved pulse will coincide with the cusp of the phase modulation at the point where the chirp imposed is almost linear, yielding a flatter spectral profile. This process can be interpreted in terms of time-to-frequency mapping [9]: if the chirping is sufficiently large, the envelope of the optical spectrum will become a scaled replica of the intensity pulse [10]. In many applications, increasing the bandwidth of the combs is also desirable. However, the bandwidth of the combs generated in this IM-PM scheme is limited due to the bandwidth and RF power-handling capability of the PM. Of course, by placing several phase modulators in tandem, larger bandwidths can be achieved (see, e.g. [11, 12]). Another method is by first compressing the comb to a short pulse and then using nonlinear propagation in dispersion decreasing or highly nonlinear fiber (HNLF) to broaden the spectrum [13-15].

Our group has also demonstrated a method that achieved simultaneous bandwidth enhancement and improved flatness by exploiting four-wave mixing (FWM) in a highly nonlinear fiber (HNLF) [16]. In this method, two optical frequency combs centered at different frequencies, $\omega_{1}$ and $\omega_{2}$ (with $\omega_{2}>$ $\left.\omega_{1}\right)$ are mixed in a HNLF. Initially, each comb has narrow bandwidth and poor flatness. However, after traveling through a cascade of FWM processes, new frequency combs can be generated exhibiting enhanced spectral width and flatness. For example, when properly phase-matched, the complex envelope of the Nth-order higher-frequency sideband centered at $(\mathrm{N}+1) \omega_{2}-\mathrm{N} \omega_{1}$ will be proportional to

$$
\left[\mathrm{e}_{2}(\mathrm{t})\right]^{\mathrm{N}+1}\left[\mathrm{e}_{1}{ }^{*}(\mathrm{t})\right]^{\mathrm{N}}
$$

where $e_{1}(t)$ and $e_{2}(t)$ denote the complex field envelopes of the seed combs centered at $\omega_{1}$ and $\omega_{2}$, respectively. The frequency converted signal will display an equivalent modulation index increased by a factor of $2 \mathrm{~N}+1$, provided the phase conjugation in $\mathrm{e}_{1}(\mathrm{t})$ is properly managed [16].

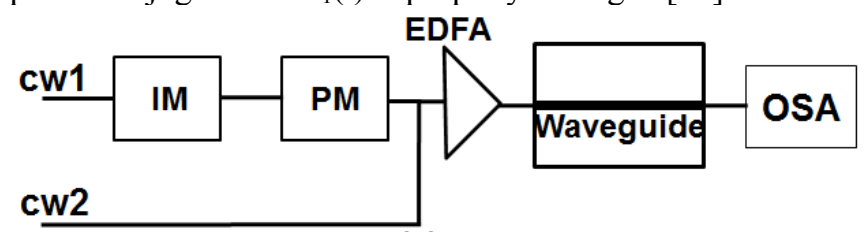

(a)

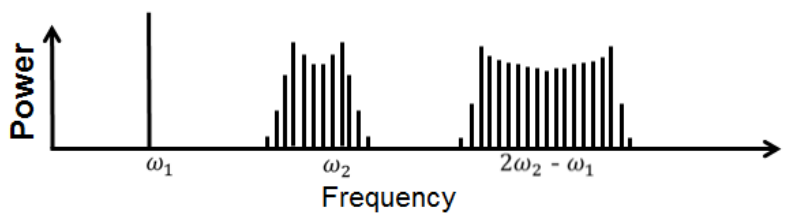

(b)

Fig. 1 (a) Experimental setup. CW-continuous wave laser, IM Intensity modulator, PM - Phase modulator, EDFA - High power erbium-doped fiber amplifier, OSA -optical spectrum analyzer (b) Bandwidth scaling of the CW comb. 
Recently, several groups have investigated nonlinear processes in silicon waveguides, such as parametric amplification [17], Raman amplification [18], and FWM [19], which provide very high nonlinearity in a chip-based geometry. These devices have proven to be practical in optical signal processing applications which require broadband wavelength conversion [20-22]. In this report, we utilize on-chip FWM to introduce a more compact and flexible scheme for spectral broadening of a frequency comb. This technique, based on principles similar to [16], allowed us to achieve $>100$ lines at $10-\mathrm{GHz}$ spacing within a $5-\mathrm{dB}$ bandwidth.

Figure 1 shows the experimental setup. This setup differs from the setup of ref. [16] in three aspects. First, the $100 \mathrm{~m}$ of HNLF is replaced by a silicon nano-waveguide, which is only $1 \mathrm{~cm}$ in length. Second, the dispersion of the silicon chip can be tailored by engineering the waveguide crosssection geometry which in turn increases the conversion bandwidth $[20,21]$. Finally, the signal centered at $\omega_{2}$ will not be modulated but instead combined with the $1^{\text {st }} \mathrm{CW}$ directly. In this case, the initial fields are given by, $\left.\mathrm{e}_{2}(\mathrm{t}) \sim \mathrm{a}_{2}(\mathrm{t}) \exp [\mathrm{j} \phi(\mathrm{t}))\right]$ and $\mathrm{e}_{1}(\mathrm{t}) \sim 1$, which after mixing produce a new first order FWM component at $2 \omega_{2}-\omega_{1}$ given by $e_{2}{ }^{2}(t) e_{1}{ }^{*}(t)=a_{2}(t)^{2} \exp [2 j \phi(t)]$. This indicates the new comb's bandwidth is roughly doubled with respect to the signal centered around $\omega_{2}$. Compared to ref. [16], this setup is more compact and flexible, although it comes at the expense of a reduction in the achievable bandwidth scaling. The reason behind these differences is that in [16], the signal centered at $\omega_{1}$ first goes through an IM and is then combined with the second $\mathrm{CW}$ input centered at $\omega_{2}$, after which both signals enter the PM followed by a dispersive element. The role of the dispersive element was to delay the modulated signal by half an RF period. This delay effectively performs a $\pi$ phase shift to the field envelope term $\mathrm{e}_{1}{ }^{*}(\mathrm{t})$ in Eq. (1) to ensure that the phase modulations on the two fields are additive during the FWM process rather than acting to cancel. Although this approach will effectively enhance the bandwidth by a factor of three for first order mixing terms (50\% higher than achieved with our current scheme), the dispersion must be changed in accord with the changes in the comb repetition rate. Therefore, repetition rate tunability is compromised.

Our silicon waveguide was patterned with electron-beam lithography on a silicon-on-insulator wafer. We designed the cross-section geometry so that it has a zero-group-velocity dispersion (GVD) wavelength within the C-band for quasiTM polarization. The geometry is $800 \mathrm{~nm}$ in width by 250 $\mathrm{nm}$ in thickness. The two ends of the waveguide are inversely tapered to $100 \mathrm{~nm}$ allowing light to be coupled in and out through a fiber taper. The end of the waveguide is followed by a U-groove to stabilize the fiber for high-power applications. The above setup provides a fiber-to-fiber loss of $-8 \mathrm{~dB}$ which includes the coupling and propagation losses. The propagation loss for our silicon waveguide is estimated to be about $3.5 \mathrm{~dB} / \mathrm{cm}$ [23]. Finally, the nonlinear parameter is calculated to be in the order of $10^{-1}[\mathrm{rad} /(\mathrm{W} \cdot \mathrm{cm})]$, three orders of magnitude higher than the HNLF in [16].
We first test our waveguide with $\mathrm{CW}$ input for both pump and probe; the measured FWM spectrum at the nanowaveguide's output is given in Fig. 2(a), which shows a -19 $\mathrm{dB}$ conversion efficiency (defined as the idler to probe power ratio at the chip's output) when the pump power is $200 \mathrm{~mW}$. At this power level, the waveguides sometimes get damaged after several hours of operation. We also measured the conversion efficiency spectrum of this waveguide. The result for pumping at $1550 \mathrm{~nm}$ is given in Fig. 2(b). We can achieve a relatively broad and flat conversion efficiency $(>100 \mathrm{~nm}$, $<5 \mathrm{~dB}$ variation) when pumping at $1550 \mathrm{~nm}$. Since the FWM conversion bandwidth is inversely proportional to the square root of the product of the interaction length and the GVD [24], this means that the zero dispersion point of the waveguide is very close to $1550 \mathrm{~nm}$. The measurement limit in these experiments is given by the tuning range of the probe laser (1460-1580 nm). In our context, this means that the two frequency combs can be placed significantly further apart and generate broader combs operating at high repetition rates. It is worth noting that simultaneous broadband and flat conversion efficiency can be achieved for any pumping wavelength within the EDFA gain bandwidth, allowing our input frequency to be chosen anywhere within this range.

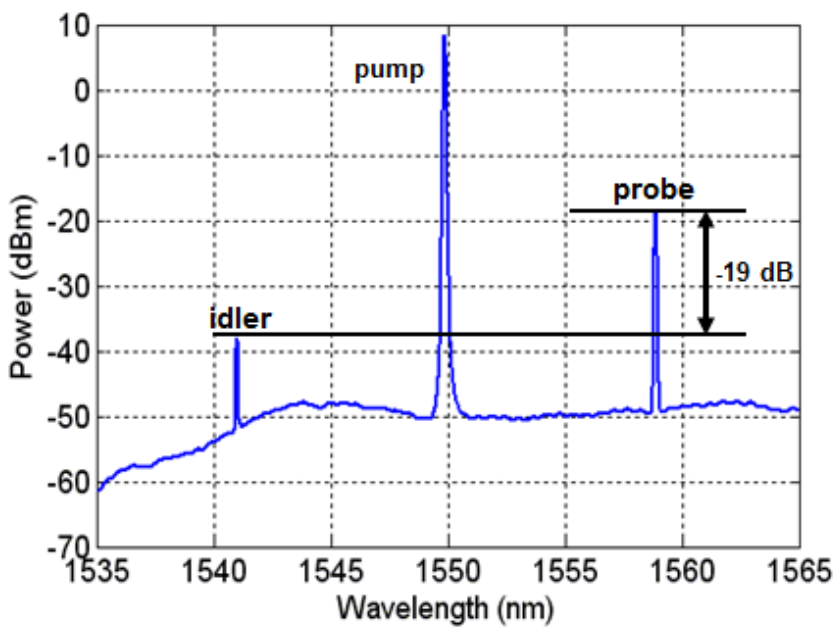

(a)

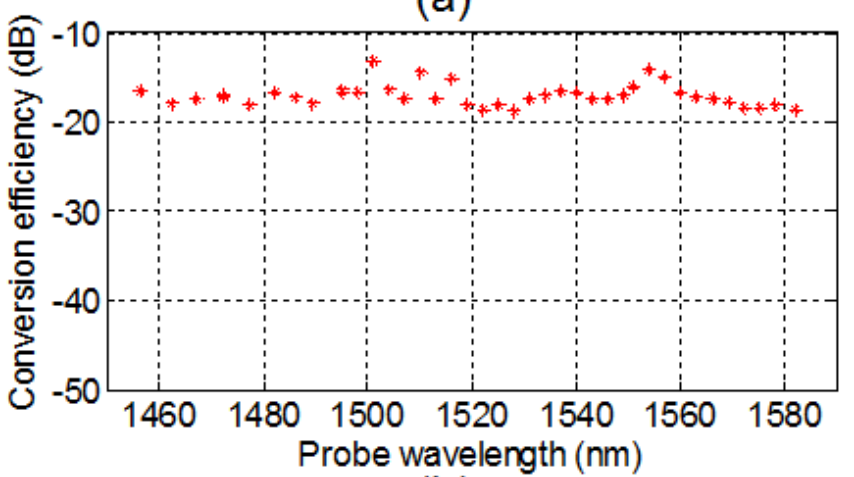

(b)

Fig. 2 (a) Spectrum for the FWM with CW inputs, (b) FWM conversion efficiency spectrum pumping at $1550 \mathrm{~nm}$

We demonstrate our technique using a high-power frequency-tunable electro-optic comb source, which is comprised of 3 phase modulators and 1 intensity modulator. 
The performance details of this light source can be found in [12]. Our FWM results are shown in Fig. 3. The input frequency comb is centered at $1545 \mathrm{~nm}$ with $10 \mathrm{GHz}$ repetition rate and is comprised of about 55 lines at $5-\mathrm{dB}$ bandwidth, as shown in Fig. 3(a). The input unmodulated $\mathrm{CW}$ is centered at $1560 \mathrm{~nm}$. As expected, the FWM generated near $1530 \mathrm{~nm}$ has more than 100 lines in a $5 \mathrm{~dB}$ bandwidth. The small asymmetry and dips visible in the FWM term are likely caused by the conversion efficiency variations as indicated in Fig. 1(b). This could be optimized by a more careful engineering of waveguide dispersion.

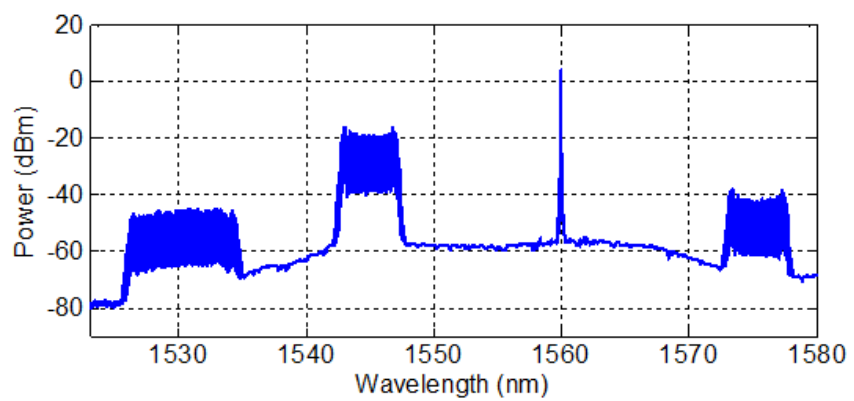

(a)

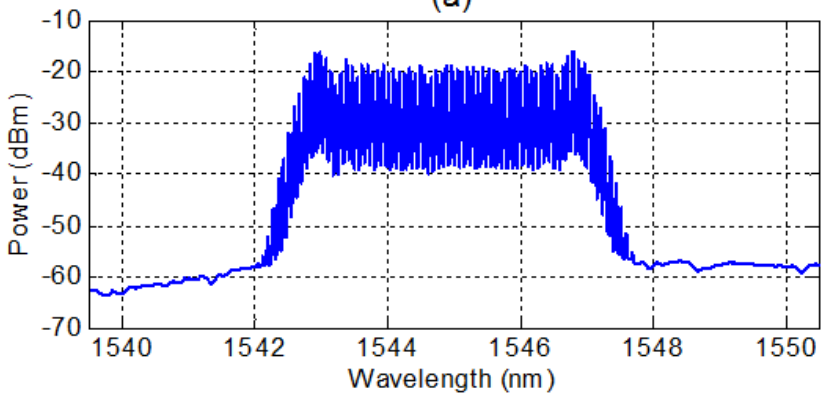

(b)

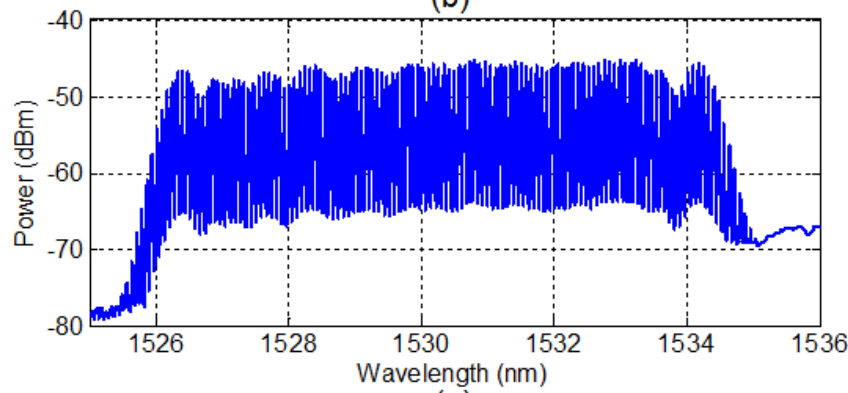

(c)

Fig. 3 (a) Output spectrum of the waveguide (b) and (c) Zoom in view of initial comb spectrum and generated comb after FWM interaction, respectively.

We now illustrate the wavelength-tuning capabilities of the broadened FWM combs. Here, the seed is instead synthesized using a simpler comb generator consisting of a single IM and single PM with $10 \mathrm{GHz}$ repetition rate (spectrum shown in Fig. 4(a)). The wavelength separation between the $\mathrm{CW}$ and seed comb is continuously varied keeping their wavelengths centered near $1550 \mathrm{~nm}$ $\left(\left(\lambda_{1}+\lambda_{2}\right) / 2 \approx 1550 \mathrm{~nm}\right)$. Fig. 4(b)-(d) illustrates the first generated FWM sideband when the spacing between the input $\mathrm{CW}$ waveform and seed comb is set to $5 \mathrm{~nm}, 10 \mathrm{~nm}$ and $20 \mathrm{~nm}$, respectively. The bandwidth of the comb generated through FWM is again roughly twice that of the seed comb and remains approximately constant as it is tuned.

In summary, we have demonstrated a simple on-chip scheme to scale the bandwidth of a phase and intensity modulated $\mathrm{CW}$ comb. Compared to the previous nonlinear optical fiber scheme [16], our generated combs exhibit comparable bandwidth $(>1 \mathrm{THz})$ and good flatness $(>100$ lines within 5-dB bandwidth). What sets our scheme apart is the added flexibility and compactness.
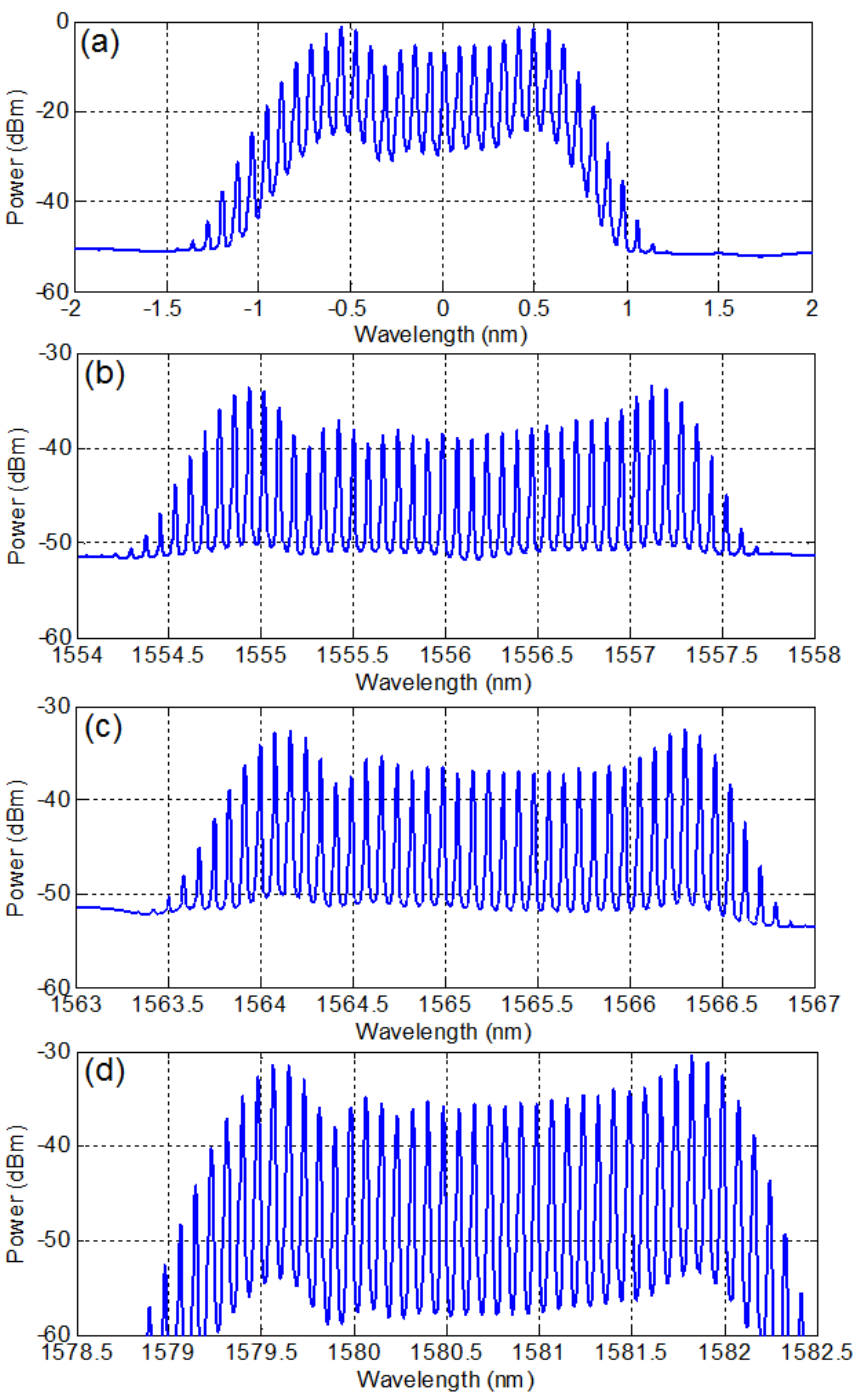

Fig. 4 Wavelength tuning of FWM generated frequency combs. (a) Input spectrum of the PM-IM comb. Output spectra of comb with different wavelength separation between $2 \mathrm{CW}$ lasers. The wavelength separation of the two inputs are $5 \mathrm{~nm}, 10 \mathrm{~nm}$ and $20 \mathrm{~nm}$, for (b)-(d) respectively.

\section{Acknowledgements}

This project was supported in part by the Naval Postgraduate School under grant N00244-09-1-0068 and by the National Science Foundation under grant ECCS-0925759. Victor Torres-Company gratefully acknowledges funding from the Swedish Research Council (VR). 


\section{References}

[1] H. Murata, A. Morimoto, T. Kobayashi, and S. Yamamoto, "Optical pulse generation by electrooptic-modulation method and its application to integrated ultrashort pulse generators," IEEE J. Sel. Top. Quantum Electron. 6, 1325-1331 (2000).

[2] V. Torres-Company, and A. M. Weiner, "Optical frequency comb technology for ultra-broadband radio-frequency photonics", Laser \& Photon. Rev. 8, 368-393 (2014).

[3] T. Ohara, H. Takara, T. Yamamoto, H. Masuda, T. Morioka, M. Abe, and H.Takahashi, "Over 1000-channel ultradense WDM transmission with supercontinuum multicarrier source", J. Lightwave Technol. 24, 2311-2317 (2006).

[4] V. R. Supradeepa, Christopher M. Long, Rui Wu, Fahmida Ferdous, Ehsan Hamidi, Daniel E. Leaird and Andrew M. Weiner, "Comb-based radiofrequency photonic filters with rapid tunability and high selectivity," Nature Photon. 6, 186-194 (2012)

[5] Z. Jiang, C. B. Huang, D. E. Leaird, and A. M. Weiner, "Optical arbitrary waveform processing of more than 100 spectral comb lines," Nature Photon., 1, 463-467 (2007).

[6] M. Fujiwara, M. Teshima, J. Kani, H. Suzuki, N. Takachio, and K. Iwatsuki, "Optical Carrier Supply Module Using Flattened Optical Multicarrier Generation Based On Sinusoidal Amplitude and Phase Hybrid Modulation,” J. Lightwave Technol. 21, 2705-2714 (2003).

[7] T. Otsuji, M. Yaita, T. Nagatsuma, E. Sano, "10-80-Gb/s highly extinctive electrooptic pulse pattern generation," IEEE J. Select. Top. Quantum Electron. 2, 643-649 (1996).

[8] B. H. Kolner and M. Nazarathy, "Temporal imaging with a time lens," Opt. Lett. 14, 630-632 (1989)

[9] J. Azaña, "Time-to-frequency conversion using a single time lens," Opt. Commun. 217, 205-209 (2003).

[10] V. Torres-Company, J. Lancis, and P. Andres, "Lossless equalization of frequency combs," Opt. Lett. 33, 1822-1824 (2008).

[11] A. Ishizawa, T. Nishikawa, A. Mizutori, H. Takara, H. Nakano, T. Sogawa, A. Takada, and M. Koga, "Generation of 120-fs laser pulses at 1$\mathrm{GHz}$ repetition rate derived from continuous wave laser diode," Opt. Express 19, 22402-22409 (2011).

[12] A. J. Metcalf, V. Torres-Company, D. E. Leaird, A. M. Weiner, "HighPower Broadly Tunable Electrooptic Frequency Comb Generator", IEEE J. Sel. Top. Quantum Electron. 84, 3500306, (2013)

[13] C.B. Huang, S.G. Park, D.E. Leaird, and A.M. Weiner, "Nonlinearly Broadened Phase-Modulated Continuous-Wave Laser Frequency Combs Characterized using DPSK Decoding," Opt. Express 16, 2520-2527 (2008).

[14] R. P. Scott, N. K. Fontaine, J. P. Heritage, B. H. Kolner, and S. J. B. Yoo, "3.5-THz Wide, 175 Mode Optical Comb Source", in Optical Fiber Communication Conference and Exposition and The National Fiber Optic Engineers Conference, OWJ3 (2007).

[15] B. P.-P. Kuo, E. Myslivets, V. Ataie, E. G. Temprana, N. Alic, and S. Radic, "Wideband Parametric Frequency Comb as Coherent Optical Carrier," J. Lightwave Technol. 31, 3414-3419 (2013).

[16] V. R. Supradeepa, and A. M. Weiner, "Bandwidth scaling and spectral flatness enhancement of optical frequency combs from phase-modulated continuous-wave lasers using cascaded four-wave mixing," Opt. Lett. 37, 3066-3068 (2012)

[17] M. A. Foster, A. C. Turner, J. E. Sharping, B. S. Schmidt, M. Lipson and A. L. Gaeta. "Broad-band optical parametric gain on a silicon photonic chip," Nature 441, 960-963 (2006).

[18] Claps, R., Dimitropoulos, D., Raghunathan, V., Han, Y. \& Jalali, B. "Observation of stimulated Raman amplification in silicon waveguides." Opt. Express 11, 1731-1739 (2003).

[19] H. Fukuda, K. Yamada, T. Shoji, M. Takahashi, T. Tsuchizawa, T. Watanabe, J. Takahashi, and S. Itabashi, "Four-wave mixing in silicon wire waveguides," Opt. Express 13, 4629-4637 (2005).

[20] A. C. Turner-Foster, M. A. Foster, R. Salem, A. L. Gaeta, and M. Lipson, "Frequency conversion over two-thirds of an octave in silicon nanowaveguides," Opt. Express 18, 1904-1908 (2010).

[21] R. K. W. Lau, M. Menard, Y. Okawachi, M. A. Foster, A. TurnerFoster, R. Salem, M. Lipson, and A. L. Gaeta, "Continuous-wave midinfrared frequency conversion in silicon nanowaveguides," Opt. Lett. 36, 1263-1265, (2011).

[22] H. Ji, M. Pu, H. Hu, M. Galili, L. K. Oxenlowe, K. Yvind, J. M. Hvam and P. Jeppesen,, "Optical waveform sampling and error-free demultiplexing of $1.28 \mathrm{~Tb} / \mathrm{s}$ serial data in a nanoengineered silicon waveguide," $J$. Lightwave Technol. 29, 426-431, (2011)
[23] H. Shen, L. Fan, J. Wang, J. C. Wirth, and M. Qi, "A Taper to Reduce the Straight-to-Bend Transition Loss in Compact Silicon Waveguides", IEEE Photon. Tech. Lett. 22, 1174-1176, (2010).

[24] M. E. Marhic, N. Kagi, T. K. Chiang, and L. G. Kazovsky, "Broadband fiber optical parametric amplifiers," Opt. Lett. 21, 573-575 (1996). 